LETTERS TO THE EDITOR

\title{
A Note on Evolutionary Stable Strategies and Game Dynamics
}

In 1974 J. Maynard Smith introduced the fundamental notion of an evolutionarily stable strategy (ESS) in order to explain the evolution of genetically determined social behaviour within a single animal species. If the possible pure strategies for contests within a species are $1,2, \ldots, n$, and if $A=\left(a_{i j}\right)$ is the payoff matrix, then $a_{i j}$ is the payoff for the pure strategy $i$ played against the pure strategy $j ; \sum_{j} a_{i j} q_{j}$ is the payoff for the pure strategy $i$ against the mixed strategy given by a probability vector $q=\left(q_{1}, \ldots, q_{n}\right)$; and

$$
p A q=\sum_{i, j} p_{i} a_{i j} q_{j}
$$

the payoff for strategy $p=\left(p_{1}, \ldots, p_{n}\right)$ played against $q$. Let

$$
S_{n}=\left\{x=\left(x_{1}, \ldots, x_{n}\right): \sum_{j} x_{j}=1, \quad x_{i} \geqslant 0 \quad \text { for } i=1, \ldots, n\right\}
$$

be the simplex of all possible strategies.

D1: (Maynard Smith, 1974) A state $p \in S_{n}$ is called an ESS if for all $q \neq p$ either $p A p>q A p$ or $p A p=q A p$ and $p A q>q A q$.

In Taylor \& Jonker (1978) the authors used the fact that the payoff, in animal contests, corresponds "by definition" to the rate of increase. This suggests for the investigation of the evolution of behaviour the dynamical model given by

$$
\dot{x}_{i} / x_{i}=\sum_{j} a_{i j} x_{j}
$$

With this equation, however, the strategies $\left(x_{1}, \ldots, x_{n}\right)$ don't remain on the simplex. But since only the differences in payoff are relevant for the game, one may consider

$$
\dot{x}_{i} / x_{i}=\sum_{j} a_{i j} x_{j}-A,
$$

where the function $A$ is chosen in such a way that $\sum_{i} \dot{x}_{i}=0$ whenever 
$\sum_{i} x_{i}=1$. This leads to the equation

$$
\dot{x}_{i}=x_{i}\left(\sum_{j} a_{i j} x_{j}-\sum_{i, j} x_{i} a_{i j} x_{j}\right) \quad i=1, \ldots, n
$$

on the (invariant) simplex $S_{n}$.

Having derived this equation, Taylor \& Jonker proceed to show, as their main result, that if $p$ is an ESS satisfying a mild regularity condition, then $p$ is an equilibrium state for (1) which is strictly stable (i.e. all eigenvalues have strictly negative real part). The converse is not valid.

In this note, we give a simple characterization of ESS which implies immediately that every ESS is an equilibrium state for (1) which is asymptotically stable (all orbits near $p$ converge to $p$ ). Thus both the hypothesis and the result are slightly weaker than in Taylor \& Jonker (1978). We begin by reformulating D1.

D2: A state $p \in S_{n}$ is called an ESS if for all $q \in S_{n}$ one has

$$
p A((1-\varepsilon) p+\varepsilon q)>q A((1-\varepsilon) p+\varepsilon q)
$$

provided $\varepsilon>0$ is sufficiently small.

The equivalence of the two definitions is easily seen since (2) means

$$
(1-\varepsilon)(p A p-q A p)+\varepsilon(p A q-q A q)>0 .
$$

The interpretation of D2 is easy: if a mutation in a population with strategy $p$ introduces a small population with strategy $q$, then the $p$-population fares better than the q-population against the new (mixed) population $(1-\varepsilon) p+\varepsilon q$. D2 then is equivalent to D3: A state $p \in S_{n}$ is called an ESS if

$$
p A x>x A x
$$

for all $x \in S_{n}, x \neq p$, in a sufficiently small neighbourhood of $p$.

This follows by multiplying (2) by $\varepsilon$ and adding $(1-\varepsilon) p A((1-\varepsilon) p+\varepsilon q)$ on both sides.

Let us now use the fact that $p$ is the unique maximum of the function

$$
P:\left(x_{1}, \ldots, x_{n}\right) \rightarrow \prod_{i} x_{i}^{p_{i}}
$$

on $S_{n}$, since the Lagrange multiplier must be equal to

$$
\partial P / \partial x_{i}=P\left(P_{i} / x_{i}\right)
$$


which implies that the $x_{i}$ must be proportional (and hence equal) to the $p_{i}$, at the maximum. The time derivative of $P$ along the orbits of $(1)$ is

$$
\begin{aligned}
\dot{P} & =\sum_{i} \partial P / \partial x_{i} \cdot x_{i}=P \sum_{i} p_{i}\left(\sum_{j} a_{i j} x_{j}-\sum_{i, j} x_{i} a_{i j} x_{j}\right) \\
& =P(p A x-x A x) .
\end{aligned}
$$

Hence D3 is equivalent to having $\dot{P}(x)>0$ for all $x \neq p$ in a small neighbourhood of $p$. Thus

D4: A state $p \in S_{n}$ is an ESS if

$$
V=\prod_{i} p_{i}^{p_{i}}-\prod_{i} x_{i}^{p_{i}}
$$

is a strict local Ljapunov function at $p$ for (1).

As a corollary of D4, one obtains that every ESS is asymptotically stable. As an example, we mention that for the case of the "4-hypercycle", where

$$
A=\left[\begin{array}{cccc}
0 & k_{1} & 0 & 0 \\
0 & 0 & k_{2} & 0 \\
0 & 0 & 0 & k_{3} \\
k_{4} & 0 & 0 & 0
\end{array}\right]
$$

(with $k_{i}>0$ ) the unique equilibrium in the interior of $S_{n}$ is always globally stable; the function $V$ is a Ljapunov function if all $k_{i}$ are equal, but it is never a strict Ljapunov function. For the "3-hypercycle", the equilibrium is again always a global attractor, but $V$ is a (strict) Ljapunov function if the three constants $\sqrt{k_{1}}, \sqrt{k_{2}}$ and $\sqrt{k_{3}}$ satisfy the (strict) triangle inequality.

These examples imply that not every asymptotically stable equilibrium is an ESS, and that (as noted already by Haigh in an appendix to Maynard Smith (1974) even for $n=3$ there need not be an ESS at all. Another example is given by

$$
A=\left[\begin{array}{rrr}
0 & 1 & -1 \\
-1 & 0 & 1 \\
1 & -1 & 0
\end{array}\right]
$$

(which corresponds to the game of scissors-paper-stone which is treated by a different ecological model in May \& Leonard (1975). Here $p=(1 / 3,1 / 3,1 / 3)$ and $\dot{P}=0$ on $S_{3}$ (every orbit in the interior is periodic).

Since there is nothing special about the Ljapunov function $V$, it could be that under certain circumstances it would be more appropriate to study asymptotically stable equilibria of (1), rather than ESS. 
Let us mention finally that equation (1), and relatives of it, have been investigated in Eigen (1971), Eigen \& Schuster (1978) and Schuster, Sigmund, Wolff \& Hofbauer (1978) in the context of self organization of macromolecules. It seems interesting that the same equation (1) plays a certain role at the two very ends, so to speak, of evolution, namely prebiotic evolution and evolution of animal behaviour.

Recently we learned that Zeeman had already obtained a proof that every ESS is an attractor. His result has not been published yet. We would like to thank Professors Maynard Smith and Zeeman for helpful advice and making unpublished material available to us.

\section{REFERENCES}

EIGEN, M. (1971). Self-organization of matter and the evolution of biological macromolecules. Die Naturwissenschaften $\mathbf{5 8 , 4 6 5 .}$

EIGEN, M. \& SCHUSTER, P. (1978). The hypercycle, a principle of natural self-organization. Part B. The abstract hypercycle. Die Naturwissenschaften 65, 7.

MAY, R. \& LeONARD, W. (1975). Nonlinear aspects of competition between three species, SIAM. J. app. Math. 29, 243.

MAYNARD SMITH, J. (1974). The theory of games and the evolution of animal conflicts. J. theor. Biol. 47, 209.

TAYLOR, P. \& JONKER, L. (1978). Evolutionary stable strategies and game dynamics. Math. Biosci. 40, 145.

Schuster, P., Sigmund, K. \& WolfF, R. (1979). On $\omega$-limits for competition between three species, SIAM. J. app. Math. 37.

Schuster, P., Sigmund, K. \& WolfF, R. (1978). Dynamical systems under constant organization, Part I: A model for catalytic hypercycles. Bull. Math. Biophys. 40, 743.

Part II (with J. Hofbauer): Homogeneous growth functions of degree $\mathrm{p}=2$, to appear in SIAM. J. app. Math.

Part III: Cooperative and competitive behaviour of hypercycles, $J$. Diff. Equs. 32, 357.

ZEEMAN, E. C. Population dynamics from game theory-preprint.

University of Vienna, Vienna

Austria

(Received 9 May 1979)
J. HOFBAUER, $\uparrow$ P. SCHUSTER $\ddagger$ AND K. SIGMUND $\dagger$

$\dagger$ J. Hofbauer and K. Sigmund, Institut für Mathematik.

$\ddagger$ P. Schuster, Institut für Theoretische Chemie und Strahlenchemie Universität Wien. 